\title{
A COMPARATIVE STUDY IN THE DIABETES MELLITUS PATIENTS FOR ORAL MANIFESTATION AT TERTIARY CARE HOSPITAL IN NORTH KARNATAKA
}

\author{
ANAND V NIMBAL*, VIKAS C DESAI, SHARDHA BAI RATHOD \\ Department of Dentistry, BLDEU's Shri B.M. Patil Medical College and Research Centre, Vijayapura, Karnataka, India. \\ Email: dranandnimbal@gmail.com
}

Received: 13 August 2016, Revised and Accepted: 31 August 2016

\section{ABSTRACT}

Objective: Diabetes mellitus is the leading health problem across the world. It is associated with several complications such as retinopathy, neuropathy, oral manifestations, chronic macrovascular and microvascular complications. Our objective is to compare these oral manifestations in controlled and uncontrolled diabetic patients.

Methods: A study was conducted on total of 100 diabetic patients. The patients were divided into two groups: Group I (n=50) consists of controlled diabetic patients and Group II $(n=50)$ consists of uncontrolled diabetic patients. Before, the start of the study proper oral examination was done for both the groups. Samples were collected and were subjected to microbial examination. Comparison between both the groups was done for oral manifestations.

Results: It showed that uncontrolled diabetic patients had a higher incidence of oral manifestations such as hyposalivation, parotid enlargement, burning sensation of mouth, taste alterations, sialorrhea, dental carries, and microbial lesions than controlled diabetes patients.

Conclusion: Uncontrolled diabetic patients had higher risk of oral manifestations than controlled diabetic individuals.

Keywords: Diabetes mellitus, Retinopathy, Neuropathy, Oral manifestations, Periodontitis, Hyposalivation.

(C) 2016 The Authors. Published by Innovare Academic Sciences Pvt Ltd. This is an open access article under the CC BY license (http://creativecommons. org/licenses/by/4. 0/) DOI: http://dx.doi.org/10.22159/ajpcr.2016.v9s3.14696

\section{INTRODUCTION}

Diabetes mellitus is progressing at an alarming rate worldwide. It is associated with several micro- and macro-vascular complications [1] Interestingly periodontal infections are more commonly observed in diabetic individuals [2]. Diabetes negatively affects the salivary gland function and immune function which further increases the risk of oral infections in these individuals $[3,4]$. Oral infections including hyposalivation or xerostomia which is associated with burning sensation of mouth, loss of sensation of taste, enlargement of salivary glands, candida infections, and few autoimmune lesion such as lichen planus are reported [1]. Hence, this study was conducted to identify the risk of oral infections in controlled and uncontrolled diabetic individuals.

\section{METHODS}

Among 150 Type 2 diabetic patients from various tertiary care hospitals present in and around Vijaypur, Karnataka, a total of 100 patients were randomly selected with no other severe health problems. They were divided into two groups. Group I consist of 50 controlled diabetic patients who were stabilized with insulin, oral hypoglycemic or controlled diet. Group II consists of 50 patients with uncontrolled diabetes and without dietary control. Special care is taken for maintaining medical records with previous medical history and habits.

Before conducting the study, ethical clearance was obtained from Ethics Committee. The official permission for examination was obtained from all the concerned authorities. Voluntary written informed consent was obtained from patients participating in the study before the clinical examination.

Proper oral examination of the oral cavity was done with adequate natural light using plain mouth mirror and WHO probe. Examination of dental caries, periodontal problems, oral infective lesion such as candidiasis, herpes, glossitis, cheilitis angular other benign neoplastic lesion, and proliferative lesions such as leukoplakia erythroplakia, and autoimmune lesion such as lichen planus.

Examination for the parotid enlargement, hyposalivation, taste alterations, and burning sensations of mouth. The oral swab sample is taken from buccal mucosa, dorsum of the tongue using sterile cotton swabs. The samples are sent to microbiology lab for culture. The sabouraud dextrose agar medium is taken for the cultural growth of the candida. Subculturing is done to differentiate morphological colonies.

\section{RESULTS}

Analysis of medical records indicated that high blood pressure was observed in uncontrolled diabetic group compared to the controlled once.

Sialadenosis is an asymptomatic bilateral parotid gland enlargement commonly seen in the diabetes patients compared to the other salivary glands, in diabetic sialosis the increased volume of the glands both in acinar as well as ductal cells. This study shows that uncontrolled diabetic patients are more compared to the controlled. Hyposalivation or xerostomia, the term for the subjective sensation of the dry mouth is a common symptom in diabetics. The sensation of oral dryness is related to a reduced flow rate of both unstimulated and stimulated whole saliva. This study shows that hyposalivation is the major finding in the uncontrolled patients than controlled (Table 1).

Taste impairment the ability to detect and recognize sweet, salty, and bitter taste is impaired in both Type 1 and 2 diabetics. Many diabetics complain of burning sensation in the oral cavity. BMS have been related to increased stimulation of the capsaicin receptor by candida metabolites. Our study shows relatively more results finding for the uncontrolled once (Table 1).

A higher caries incidence is seen among diabetics as compared to nondiabetics. Both advanced periodontal diseases and dental caries 
may lead to the loss of teeth. The autonomic neuropathy can reduce salivary flow rate, which can reduce salivary flow rate which can lead to dental caries and tooth loss. This study shows relatively more results finding for the uncontrolled once (Table 2).

In both the groups on oral examination, the presence of oral mucosal lesions such as lichen planus and recurrent aphthous ulceration has been diagnosed. Erythematous lesions on the palate are observed, which is related to prosthesis, it is diagnosed as denture stomatitis associated to candidiasis is seen. This study shows the oral lesion are more in the uncontrolled compared to the controlled once (Tables 2 and 3).

\section{DISCUSSION}

Diabetes mellitus is a metabolic syndrome considered to be caused by multiple factors resulting from a deficiency of insulin, which may be absolute due to pancreatic $\beta$-cell destruction (Type 1 ) or relative due to an increased resistance of the tissues to insulin (Type 2). A series of alterations in the oral mucosa in diabetic patients have been reported including gingivitis, periodontitis, and oral mucosal diseases that favor infections such as candidiasis, salivary gland dysfunction, altered taste, glossodynia, and stomatopyrosis [1].

Pathogenic lesions in diabetic patients are not usual but few cases were reported which was not associated with age. Oral mucosa is normally protected with saliva when present in adequate quality and quantity. Saliva makes lubrication, cleansing, and $\mathrm{pH}$ buffering. It also secretes antimicrobial proteins such as secretory immunoglobulin A and aggregation and clearance of bacteria. Innate immunity in the mouth is maintained by the epithelium and oral mucosa. Hyposalivation leads to development of several oral manifestations [5].

One of the oral manifestations in diabetic patients is hyposalivation. It is due to polyuria and involvement of salivary glands. The functioning tissues in salivary glands are replaced by adipose tissue, further reduces salivary secretion and produces burning symptoms of mouth [6-9] Certain medicines such as diuretics were also associated with such symptoms. The main function of saliva is to maintain normal flora in the oral cavity. Whereas reduced saliva promotes the growth of Candida (fungi) species. In this study, also it is reported that candidiasis of

Table 1: Signs and symptoms observed in both controlled and uncontrolled diabetic patients

\begin{tabular}{lll}
\hline Signs and symptoms & Uncontrolled (\%) & Controlled (\%) \\
\hline Parotid enlargement & 58.6 & 26.3 \\
Hyposalivation & 93 & 64.1 \\
Taste alterations & 52.5 & 23.9 \\
Burning mouth sensations & 20.4 & 15.1 \\
\hline
\end{tabular}

Table 2: Presence of dental caries and periodontal diseases in both controlled and uncontrolled diabetics

\begin{tabular}{llll}
\hline Patients & $\begin{array}{l}\text { Dental } \\
\text { caries }\end{array}$ & $\begin{array}{l}\text { Patients with } \\
\text { lesions }\end{array}$ & $\begin{array}{l}\text { Periodontal } \\
\text { diseases }\end{array}$ \\
\hline Controlled & 8 & 4 & 10 \\
Uncontrolled & 24 & 20 & 29 \\
\hline
\end{tabular}

Table 3: Types of lesions observed in both controlled and uncontrolled diabetics

\begin{tabular}{lll}
\hline Lesions & Uncontrolled (\%) & Controlled (\%) \\
\hline Candidiasis & 52.4 & 16.3 \\
Herpes & 12.3 & 5.2 \\
Proliferative lesions & 29.1 & 10.6 \\
Benign neoplasias & 9.3 & 3.5 \\
\hline
\end{tabular}

predominant type is erythematous involving dentures with few cases of pseudomembranous type. These results are contradictory with previous studies, showing that healthy patients also presented with the same frequency or more candidiasis (denture sore mouth). Dentures reduce self-cleaning of palatine mucosa, may produce proliferation of fungi. Along with these, reduced vascular circulation and hygienic habits should also be considered $[10,11]$.

Farman and Nutt reported the atrophic lesions of tongue in Candida albicans infected patients [12,13]. In diabetic individual's the development candidiasis is multifactorial and not well understood, one is due to microvascularization, further reduces blood flow to tissues. The other is reduced the local resistance of the tissues.

Sialosis, the uncontrolled enlargement of bilateral parotid gland is mainly observed in diabetic patients. Our study also reported the same, the percentage of sialosis in uncontrolled diabetic patients is more in comparison to controlled diabetic individuals. In the current study, it is observed that main lesions to be varicosities of tongue and Fordyce granules which is not associate with any systemic diseases. These results were in accordance with previous studies [14].

The development of all these oral manifestations in the diabetes is multifactorial. Enhanced periodontal destruction is due to impaired neutrophil adherence, chemotaxis, and phagocytosis. Along with these high blood glucose levels in gingival cervicular fluid diminishes wound healing capacity of fibroblasts [15]. Hyperglycemia also promotes abnormal collagen metabolism, impairs proliferation of osteoblasts and weakens newly formed bone [16,17]. Advanced glycation end products (AGE) was also reported in diabetic patients. These AGE products bind with macrophage and monocytes receptors causes release of interleukin-1 and tumor necrosis factor, which further enhances tissue destruction $[15,16]$.

\section{CONCLUSION}

Oral complications - Such as hyposalivation, alteration of taste, burning sensation of mouth, parotid gland enlargement, and erythematous candidiasis - were seen in uncontrolled diabetic patients.

\section{REFERENCES}

1. Bajaj S, Prasad S, Gupta A, Singh VB. Oral manifestations in Type-2 diabetes and related complications. Indian $\mathrm{J}$ Endocrinol Metab 2012;16(5):777-9

2. Brian L, Thomas W. Periodontal disease and diabetes in young adults. J Am Med Assoc 1960;172(8):776-8.

3. Gandara BK, Morton TH. Non-periodontal oral medications of diabetes: A framework for medical care providers. Diabetes Spectr 2011;24(4):199-205.

4. Whelton H. Functions of saliva. In: Edgar M, Dawes C, O'Mullane D, editors. Saliva and Oral Health. $3^{\text {rd }}$ ed. London: British Dental Association; 2004. p. 3.

5. Dale BA, Fredericks LP. Antimicrobial peptides in the oral environment: Expression and function in health and disease. Curr Issues Mol Biol 2005;7(2):119-33.

6. Russotto SB. Asymptomatic parotid gland enlargement in diabetes mellitus. Oral Surg Oral Med Oral Pathol 1981;52(6):594-8.

7. Murrah VA, Crosson JT, Sauk JJ. Parotid gland basement membrane variation in diabetes mellitus. J Oral Pathol 1985;14(3):236-46.

8. Gibson J, Lamey PJ, Lewis M, Frier B. Oral manifestations of previously undiagnosed non-insulin dependent diabetes mellitus. J Oral Pathol Med 1990;19(6):284-7.

9. Zachariasen RD. Diabetes mellitus and xerostomia. Compendium 1992;13(4):314, 316, 318-22.

10. Odds FC. Candida and Candidiasis. $2^{\text {nd }}$ ed. London, Philadelphia, PA: Bailliére Tindall; 1988.

11. Olsen I. Denture stomatitis. Occurrence and distribution of fungi. Acta Odontol Scand 1974;32(5):329-33.

12. Farman AG, Nutt G. Oral Candida, debilitating disease and atrophic lesions of the tongue. J Biol Buccale 1976;4(3):203-26.

13. Lamey PJ, Darwaza A, Fisher BM, Samaranayake LP, Macfarlane TW, 
Frier BM. Secretor status, candidal carriage and candidal infection in patients with diabetes mellitus. J Oral Pathol 1988;17(7):354-7.

14. Birman EG, Silveira FR, Sampaio MC. Study of oral mucosal lesions in geriatric patients. Rev Fac Odontol Univ Antioq 1991;19(3):173-5.

15. Mealey BL, Oates TW; American Academy of Periodontology. Diabetes mellitus and periodontal diseases. J Periodontol 2006;77(8):1289-303.

16. Lalla RV, D'Ambrosio JA. Dental management considerations for the patient with diabetes mellitus. J Am Dent Assoc 2001;132(10):1425-32.

17. Azodo CC. Current trends in the management of diabetes mellitus: The dentist's perspective. Benign J Postgrad Med 2009;11(1):113-29. 